\section{Magnetic resonance imaging and 3-dimensional transperineal ultrasound evaluation of pelvic floor dysfunction in symptomatic women: a prospective comparative study}

\author{
Dahlia O. El-Haieg ${ }^{1}$, Nadia M. Madkour ${ }^{1}$, Mohammad Abd Alkhalik Basha², \\ Reda A. Ahmad', Somayya M. Sadek', Rania M. Al-Molla², Engy Fathy Tantwy², \\ Hosam Nabil Almassry², Khaled Mohamed Altaher², Nader E. M. Mahmoud², \\ Sameh Abdelaziz Aly ${ }^{3}$
}

Departments of ${ }^{1}$ Obstetrics and Gynecology and ${ }^{2}$ Radiodiagnosis, Zagazig University, Zagazig; ${ }^{3}$ Department of Radiodiagnosis, Benha University, Benha, Egypt

Purpose: The purpose of this study was to investigate magnetic resonance imaging (MRI) and 3-dimensional transperineal ultrasound (3D-TPUS) features of pelvic floor dysfunction (PFD) in symptomatic women in correlation with digital palpation and to define cut-offs for hiatal dimensions predictive of muscle dysfunction.

Methods: This prospective study included 73 women with symptoms suggesting PFD. 3D-TPUS, $\mathrm{MRI}$, and digital palpation of the levator ani muscle were performed in all patients. Levator hiatal antero-posterior (LHap) diameter and area ( $\mathrm{LH}$ area) were measured at rest and at maximum muscle contraction.

Results: The reduction in LHap diameter and LH area during contraction was significantly less in women with underactive pelvic floor muscle contraction (UpfmC) than in those who had normal pelvic floor muscle contraction by digital palpation $(\mathrm{P}<0.001)$. Statistically significant positive correlations $(P<0.001)$ were found between the Modified Oxford Score and 3D-TPUS and MRI regarding the reduction in the LHap diameter ( $r=0.80$ and $r=0.82$, respectively) and $\mathrm{LH}$ area $(r=0.60$ and $r=0.70$, respectively). A reduction in LHap of $<6.5 \%$ on 3D-TPUS and $<7.6 \%$ on MRI predicted UpfmC with sensitivities of $46.2 \%$ and $82.7 \%$, respectively. A reduction in $\mathrm{LH}$ area of $<3.4 \%$ on 3D-TPUS and $<3.8 \%$ on MRI predicted UpfmC with sensitivities of $75.0 \%$ and $88.5 \%$, respectively. MRI was more sensitive in detecting levator avulsion (63.4\%) than 3D-TPUS $(27.1 \%)$.

Conclusion: MRI and 3D-TPUS had strong positive correlations with findings on palpation, and at certain cut-offs for hiatal dimensions, they can be used as complementary and objective tools to improve the accuracy of diagnosis and management planning of PFD.

Keywords: Magnetic resonance imaging; Transperineal ultrasound; Pelvic floor dysfunction; Palpation

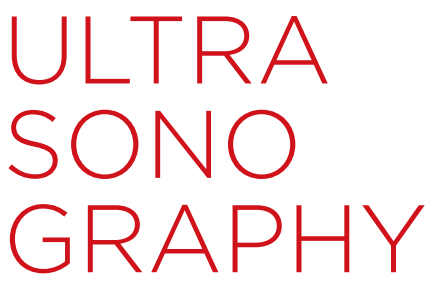

ORIGINAL ARTICLE

https://doi.org/10.14366/usg.19007 pISSN: 2288-5919 • elSSN: 2288-5943 Ultrasonography 2019;38:355-364

Received: January 16, 2019

Revised: May 1, 2019

Accepted: May 6, 2019

Correspondence to:

Mohammad Abd Alkhalik Basha, MD, Department of Radiodiagnosis, Zagazig University, Zagazig, Albalashoun, Belbiees, Alsharkia, 44631, Egypt

Tel. +20-1099098900

E-mail: Mohammad_basha76@yahoo. com

This is an Open Access article distributed under the terms of the Creative Commons Attribution NonCommercial License (http://creativecommons.org/ licenses/by-nc/4.0/) which permits unrestricted noncommercial use, distribution, and reproduction in any medium, provided the original work is properly cited.

Copyright @ 2019 Korean Society of Ultrasound in Medicine (KSUM)

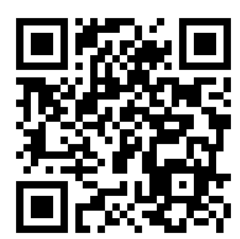

How to cite this article:

El-Haieg DO, Madkour NM, Basha MAA Ahmad RA, Sadek SM, Al-Molla RM, et al. Magnetic resonance imaging and 3-dimensional transperineal ultrasound evaluation of pelvic floor dysfunction in symptomatic women: a prospective comparative study. Ultrasonography. 2019 Oct;38(4):355-364. 


\section{Introduction}

Although the prevalence of pelvic floor dysfunction (PFD) is high, many patients have no or limited knowledge about pelvic floor health, making prevention and treatment of this condition difficult, with major negative ramifications for these patients' quality of life [1]. PFD may manifest as pelvic organ prolapse (POP), stress urinary incontinence (SUI), fecal incontinence (FI), chronic pelvic pain, sexual problems, or obstructed defecation [2].

Assessment of pelvic floor muscle (PFM) activity plays a major role in urogynecology and physiotherapy, as part of the conservative treatment of PFD [3]. Pelvic floor assessment can be done using vaginal palpation [4]. Ultrasonography (US) is used to assess PFM activity and strength, both at rest and during the Valsalva maneuver and contraction as part of the conventional urogynecological imaging protocol [5]. Three-dimensional US is a relatively lowcost, accessible, and noninvasive tool, as compared to magnetic resonance imaging (MRI) [6]. However, MRI is less operatordependent than US and has superior soft-tissue contrast resolution, which allows direct visualization of the pelvic organs and their supportive structures with dynamic sequences through a noninvasive examination [7].

Few studies have used transperineal US (TPUS) and MRI to evaluate pelvic floor muscle (PFM) (both morphological and dynamic) in patients with PFD. To date, there are no definitive cutoffs for hiatal dimensions predictive of PFD. Moreover, there is no definitive gold-standard method for diagnosing morphological and functional abnormalities of PFM. This study aimed to investigate the MRI and 3-dimensional TPUS (3D-TPUS) features of PFD in symptomatic women in correlation with digital palpation and to define cut-offs for hiatal dimensions predictive of muscle dysfunction that could benefit from surgical intervention.

\section{Materials and Methods}

\section{Study Population}

This prospective study was carried out from April 2016 to February 2018. Women complaining of one or more symptoms suggesting PFD such as POP, SUI, urgency, obstructed defecation, FI, pelvic pain, or sexual problems were recruited in the study. Women who had a history of previous genitourinary surgery, were receiving pharmacotherapy for overactive bladder, or did not understand the techniques (i.e., could not maintain holding during contraction) were excluded.

\section{Ethical Considerations}

The present study was approved by the institutional review board.
All patients were informed of the study and provided written informed consent. The study was performed in accordance with the ethical principles of the Declaration of Helsinki.

\section{D-TPUS Examinations}

All examinations were performed using a RAB6-D curved array transducer (2-8 MHz) with 3-dimensional capabilities (Voluson E6, General Electric Medical Systems, Zipf, Austria). The examinations were performed in the supine position with the hips flexed and slightly abducted after bladder emptying. The transducer was placed on the perineum, with its main axis centered in the midsagittal plane and with the application of minimal pressure, showing the inferior margin of the symphysis pubis, urethra, bladder neck, and levator ani muscle posterior to the anorectal junction (Fig. 1A). The image was adjusted with the symphysis pubis to the left and the anorectal canal to the right to identify the plane of the minimal hiatal dimensions (the shortest distance between the hyperechoic posterior border of the symphysis pubis and the hyperechoic anterior aspect of the levator ani muscle just posterior to the anorectal angle). Volumes were acquired at rest and at maximum PFM contraction. This mid-sagittal plane was then used to identify the plane of measurement in the axial plane. The line linking the midpoint of the inner edge of the symphysis pubis and puborectalis was defined as the levator hiatus axis. Tomographic US imaging was used. Eight slices with an interslice interval of $2.5 \mathrm{~mm}$ were obtained, from $5.0 \mathrm{~mm}$ below to $12.5 \mathrm{~mm}$ above the hiatal plane during maximum PFM contraction [8]. All US exams were performed by a single radiologist (with 5 years' experience with 3D-TPUS of the pelvic floor) to avoid inter-observer variability.

\section{MRI Examinations}

All MRI examinations were conducted within 2 weeks of the 3D-TPUS examinations. MRI of the pelvis was performed for all women on a 1.5-T MRI system (Achieva-class Ila, Philips Medical Systems, Best, The Netherlands) using the body phased-array coil. Static images of the pelvis (patient at rest) were first obtained in the sagittal, axial, and coronal planes using T2-weighted turbo spinecho sequences (repetition time [msec]/echo time [msec], 5,000/132; field of view, $24 \times 26 \mathrm{~cm}$; section thickness, $5 \mathrm{~mm}$; gap, $0.7 \mathrm{~mm}$; number of signals acquired, 2; matrix, $512 \times 512$; flip angle, $90^{\circ}$ ). Dynamic MRI during maximum PFM contraction was performed in the same three planes using the balanced fast-field echo sequence (5.0/1.6; field of view, $30 \times 30 \mathrm{~cm}$; gap, $0.7 \mathrm{~mm}$; section thickness, 6 $\mathrm{mm})$. The patients were asked to relax and then contract the PFM, as in the US examination, and to sustain the maximum contraction for sufficient time under the supervision of a radiologist to ensure that imaging was performed during the trial in which the maximum 
anterior abdominal wall muscle contraction was achieved. Ten images were obtained in the sagittal plane, and 22-29 images in the axial plane. Sagittal cuts were used to locate the mid-sagittal plane at the level of the symphysis pubis. Axial and coronal cuts were used to review the pelvic anatomy and any muscle defects, such as thinning, abnormal signal intensity, or avulsion.

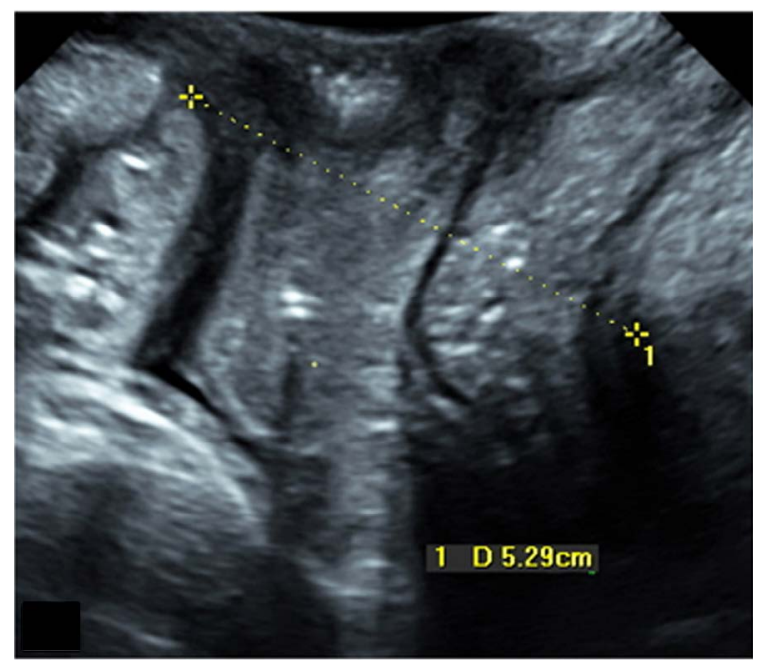

A

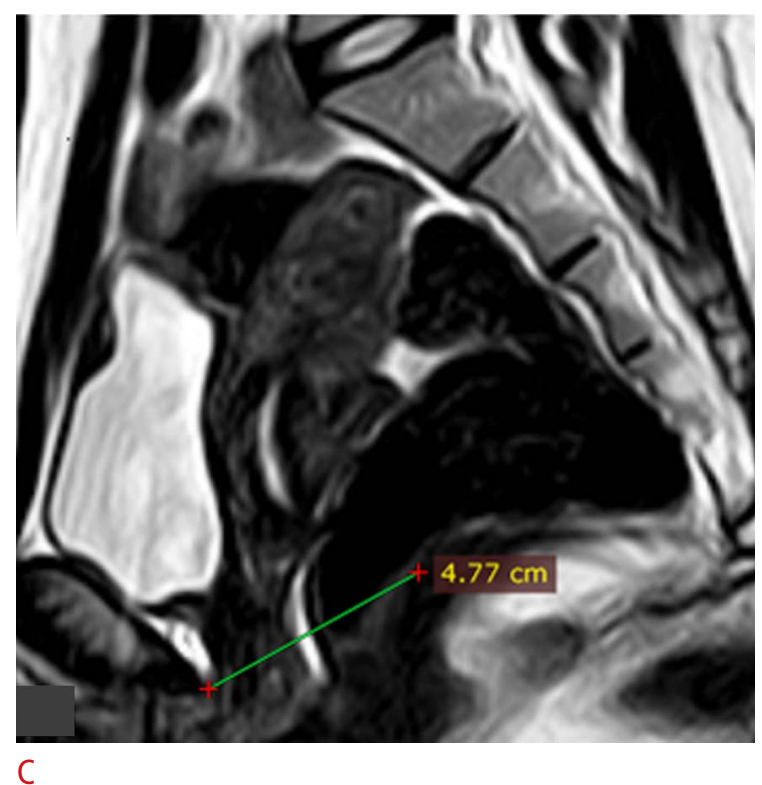

\section{D-TPUS Image Analysis}

Post-processing and offline analysis of the stored volumes were performed using the 4D view in EXT.0 software version 14 (General Electric Medical Systems). Two radiologists with 8 years of experience with 3D-TPUS independently read all US images. Any discrepancies in interpretation were resolved by a third radiologist with 10 years of experience with 3D-TPUS. All radiologists were

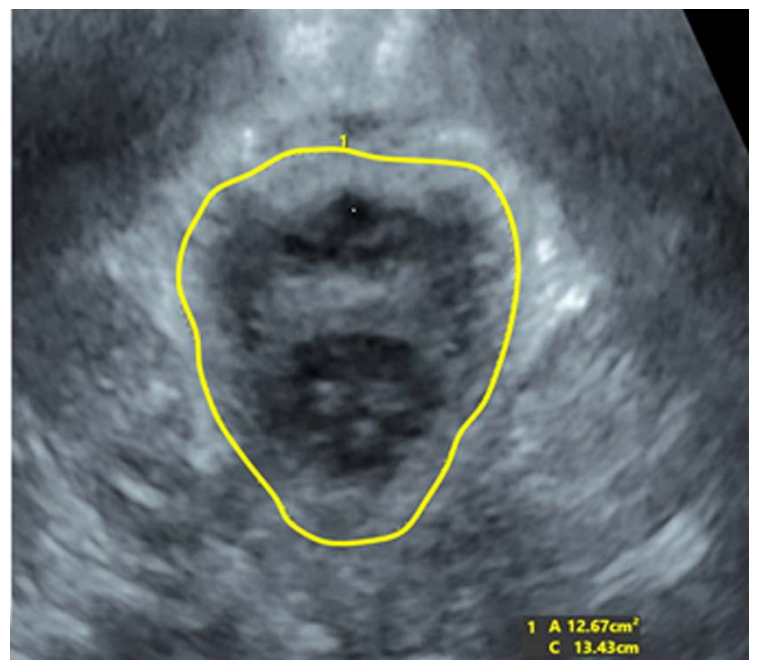

B

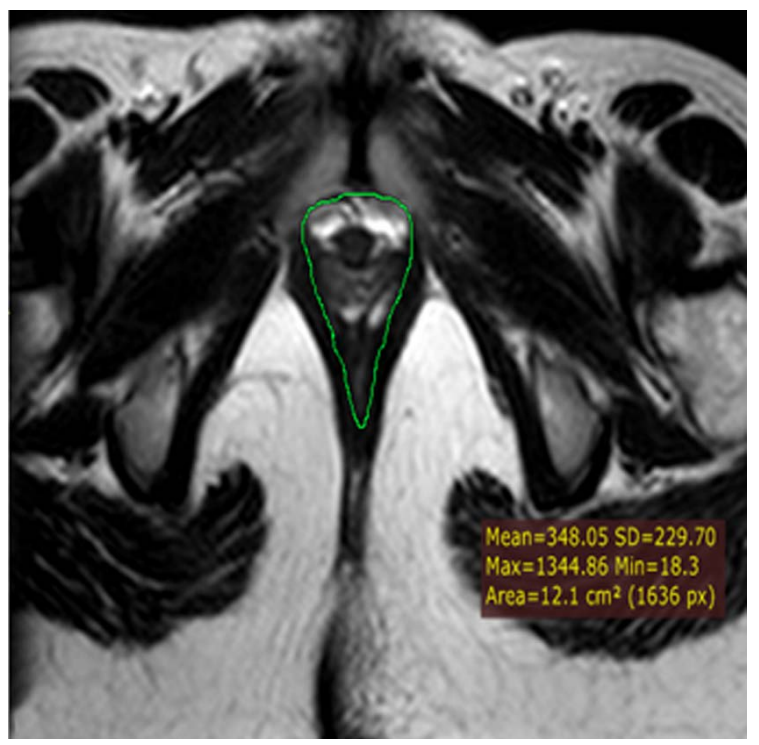

D

Fig. 1. A 33-year-old primipara woman with stress urinary incontinence.

Digital palpation revealed good contractility (Modified Oxford Score grade of 4), as well as normal pelvic floor morphology and contractility by both ultrasonography and magnetic resonance imaging (MRI). A. Sagittal 2-dimensional transperineal ultrasonography (TPUS) shows a levator hiatal antero-posterior (LHap) diameter of $5.29 \mathrm{~cm}$ at rest. B. Axial 3-dimensional TPUS reveals no muscle avulsion, with a reduction of the hiatal area to $12.67 \mathrm{~cm}^{2}$ at contraction. C. MRI sagittal cut shows a reduction in the LHap diameter to $4.77 \mathrm{~cm}$ at contraction. D. MRI axial cut shows normal signal and symmetrical thickness of both puborectalis muscles with a reduction in the hiatal area to $12.1 \mathrm{~cm}^{2}$ at contraction. 
blinded to any clinical information and the results of MRI and digital palpation. The levator hiatal antero-posterior (LHap) diameter between the inferior margin of the symphysis pubis and the puborectalis sling [9], and the LH area (the area bordered by the puborectalis muscle, the symphysis pubis, and the inferior pubic remai) [10] were measured at rest and at maximum contraction (Fig. 1B). The difference in the LHap diameter and LH area from rest to contraction and the percentage difference $\left[\left(\right.\right.$ value $_{\text {rest }}-$ value $\left._{\text {contraction }}\right) /$ value $\left._{\text {rest }}\right]$ due to contraction were calculated. Levator avulsion was defined as a clear detachment of the muscle anteromedial from the pubic bone during contraction in any of the three central slices, either unilateral or bilateral [8], as a levator ani defect or avulsion is better detected during contraction.

\section{MRI Image Analysis}

All MRI data were transferred to workstations and image analysis was performed on a dedicated platform Extended Brilliance Workstation (Philips Medical System) or PACS system (PaxeraUltima, Paxeramed, Boston, MA, USA). Two consultant radiologists with 10 years of experience with pelvic MRI independently reviewed all images, and any discrepancies in interpretation were resolved by a third consultant radiologist with 15 years of experience with pelvic $\mathrm{MRI}$. All the radiologists were blinded to any clinical information and the results of 3D-TPUS and digital palpation. LHap diameter was measured from the most inferior posterior aspect of the symphysis pubis to the anterior aspect of the hypointense signal of the puborectalis muscle (Fig. 1C). The hiatal area was measured in the axial images in the plane with the minimal hiatal dimensions (the area bordered by the symphysis pubis anteriorly and the inferior pubic rami and pubvesical muscles on both sides) at rest and at maximum contraction (Fig. 1D). The difference in the $\mathrm{LH}$ area and LHap diameter from rest to contraction was calculated using the same formula used for US to calculate the percentage difference during contraction. Avulsion of the pubovisceral component of the levator ani muscle was seen on T2-weighted images as a complete loss of connection to the pubis or as partial detachment with an apparent loss of muscle bulk, either unilaterally or bilaterally.

\section{Digital Palpation}

All patients underwent digital vaginal palpation of the puborectalis muscle. The digital palpation was performed by asking the patients to contract their PFM maximally (after education on how to contract their PFM and maintain a contraction for 3 to 10 seconds) to pull in and lift up the urethra, vagina, and rectum as if trying to control the passing of gas. The examiner put the index finger into the vagina to a maximum depth of $4 \mathrm{~cm}$ at the insertion of the puborectalis and palpated the muscle during contraction. Two well-trained gynecologists independently performed the digital palpation, and any discrepancies in interpretation were resolved by an expert panel, which consisted of three trained gynecologists, who determined the final conclusion in a consensus meeting after examination of the patient. All examiners were blinded to the results of imaging and used the validated Modified Oxford Score (MOS) [4] to grade muscle contraction on a scale of 0 to $5(0$, no contraction; 1 , minor muscle contraction ["flicker"]; 2, weak muscle contraction; 3 , moderate muscle contraction; 4, good muscle contraction; 5, strong muscle contraction against resistance). Normal pelvic floor muscle contraction (NpfmC) was defined as good or strong contraction (MOS 4 and 5) and underactive pelvic floor muscle contraction (UpfmC) was defined as weak or absent contraction (MOS 1-3).

\section{Sample Size Estimation}

The prevalence of levator ani abnormalities in patients with UpfmC was found to be $53.8 \%$ [11]. Calculating a sample size for diagnostic test characteristics is complicated by the fact that no previous studies have been published about cut-offs for $\mathrm{LH}$ area and LHap diameter measurements on US or MRI predictive of UpfmC. It was assumed that the sensitivity and specificity of US for diagnosing levator defects would be $95 \%$. Based on these expected values, we calculated that we would need 70 or more women with symptoms suggesting PFD to obtain a power of $80 \%$ and confidence of $95 \%$ to detect a significant difference in diagnostic performance with a 2-sided type I error of 5\% [12].

\section{Statistical Analysis}

All statistical analyses were conducted using SPSS version 20.0 (IBM Corp., Armonk, NY, USA). Continuous data including values for LHap diameter and LH area are presented as mean \pm SD. The MannWhitney U-test and independent-samples Student t-test were performed to analyze differences between women with UpfmC and women with NpfmC. The MOS was not normally distributed, and therefore the Spearman rank test was used to assess the correlation between digital palpation and both 3D-TPUS and MRI. An increasing rank correlation means increasing agreement between the two tests. The outcomes of the Spearman rank test range from -1 (perfect disagreement) to +1 (perfect agreement), with 0 corresponding to entirely independent rankings. Receiver operating characteristic (ROC) curve analysis was used to calculate the cut-off values and areas under the curve (AUCs) of the percentage decrease in LHap diameter and LH area on 3D-TPUS and MRI predictive of UpfmC. A $P$-value $\leq 0.05$ was considered to indicate statistical significance. 


\section{Results}

\section{Study Population}

A total of 73 women with PFD symptoms were included. Their mean age was 41.5 years (range, 23 to 67 years) and their mean body mass index (BMI) was $33.2 \mathrm{~kg} / \mathrm{m}^{2}$ (range, 23.7 to $44.3 \mathrm{~kg} / \mathrm{m}^{2}$ ). Age and BMI were significantly higher in women with UpfmC $(P<0.05)$. The majority of cases with UpfmC were in multiparous women $(98.1 \%)$, and most of them had delivered through the vaginal route (96.2\%). POP was the presenting complaint in $71 \%$ (52 of 73) of the patients. However, $60.3 \%$ (44 of 73 ) of women had more than one complaint. The demographic and clinical data of the patients are summarized in Table 1.

According to the MOS, 28.8\% (21 of 73) of the studied women had NpfmC (good or strong contraction), and $71.2 \%$ (52 of 72) had UpfmC (minor or weak contraction) (Table 2).

\section{Levator Avulsion}

Levator avulsion was diagnosed by 3D-TPUS in 20 of the 73 women (27.1\%). All patients with US-diagnosed avulsion had UpfmC based on the MOS. However, $61.5 \%$ of those with UpfmC showed no avulsion on ultrasound. MRI detected avulsion in 46 of the 73 women (63.4\%). Three patients with NpfmC based on US and the

Table 1. Demographic and clinical data of women with UpfmC versus those with $\mathrm{NpfmC}$ as diagnosed by palpation

\begin{tabular}{lccr}
\hline \multicolumn{1}{c}{ Characteristic } & UpfmC $(\mathrm{n}=52)$ & NpfmC $(\mathrm{n}=21)$ & P-value \\
\hline Age $(\mathrm{yr})$ & $43.8 \pm 11.9$ & $35.7 \pm 4.9$ & 0.003 \\
BMI $\left(\mathrm{kg} / \mathrm{m}^{2}\right)$ & $34.3 \pm 4.1$ & $30.7 \pm 4.7$ & 0.002 \\
Parity & & & 0.030 \\
$\quad$ Primipara $(\mathrm{n}=4)$ & $1(1.9)$ & $3(14.3)$ & \\
Multipara $(\mathrm{n}=69)$ & $51(98.1)$ & $18(85.7)$ & \\
Delivery mode & & & 0.008 \\
Vaginal delivery $(\mathrm{n}=65)$ & $50(96.2)$ & $15(71.4)$ & \\
Cesarean section ( $\mathrm{n}=8)$ & $2(3.8)$ & $6(28.6)$ & \\
Symptoms and signs & & & \\
Pelvic organ descent & $44(84.6)$ & $8(38.1)$ & $<0.001$ \\
SUl & $28(53.8)$ & $8(38.1)$ & 0.220 \\
Urgency & $4(7.7)$ & $8(38.1)$ & 0.001 \\
Fecal incontinence & $4(7.7)$ & 0 & 0.450 \\
Pelvic heaviness & $28(53.8)$ & 0 & $<0.001$ \\
Sexual problem & $4(7.7)$ & 0 & 0.450 \\
More than one complaint & $40(76.9)$ & $4(19.0)$ & $<0.001$ \\
\hline Values ar presented as mean & & &
\end{tabular}

Values are presented as mean \pm SD or number (\%).

UpfmC, underactive pelvic floor muscle contraction; NpfmC, normal pelvic floor muscle contraction; BMI, body mass index; SUI, stress urinary incontinence; SD, standard deviation.
MOS had unilateral avulsion on MRI (Fig. 2). However, only 17.3\% of those with UpfmC showed no avulsion on MRI (Table 3).

\section{Hiatal Dimensions}

Using both 3D-TPUS and MRI, women with UpfmC had significantly longer LHap diameters at rest and at maximum pelvic floor contraction, as well as a larger LH area than those with NpfmC. Moreover, the mean percentage decrease in the LHap and LH area during contraction was significantly less in women with UpfmC than in women with NpfmC $(P<0.001)$ as assessed by the MOS (Table 4, Figs. 2, 3). Statistically significant positive correlations were found between MOS and both 3D-TPUS and MRI findings regarding the reduction in the LHap diameter and LH area (Table 5).

\section{Cut-offs for Hiatal Dimensions}

Using ROC curves, we analyzed the data on the percentage decrease of LHap diameter and LH area on 3D-TPUS and MRI to determine cut-off values predictive of UpfmC. The ROC analyses showed that a cut-off percentage decrease in LHap diameter of $<6.5 \%$ on

Table 2. Palpation of the PFM during contraction using the MOS in the studied patients

\begin{tabular}{llcc}
\hline Contraction & Qualification & Grading & No. $(\%)(\mathrm{n}=73)$ \\
\hline UpfmC & No contraction & 0 & 0 \\
& Minor & 1 & $4(5.5)$ \\
& Weak & 2 & $16(21.9)$ \\
\multirow{3}{*}{ NpfmC } & Moderate & 3 & $32(43.8)$ \\
& Good & 4 & $21(28.8)$ \\
& Strong & 5 & 0 \\
\hline
\end{tabular}

PFM, pelvic floor muscle; MOS, Modified Oxford Score; UpfmC, underactive pelvic floor muscle contraction; NpfmC, normal pelvic floor muscle contraction.

Table 3. 3D-TPUS and MRI diagnosis of levator ani avulsion in the studied patients

\begin{tabular}{lcccc}
\hline \multicolumn{1}{c}{ Method } & UpfmC $(\mathrm{n}=52)$ & $\mathrm{NpfmC}(\mathrm{n}=21)$ & $\mathrm{X}^{2}$ & P-value \\
\hline 3D-TPUS & & & & \\
No avulsion & $32(61.5)$ & $21(100)$ & 11.10 & 0.003 \\
Unilateral avulsion & $8(15.4)$ & 0 & & \\
Bilateral avulsion & $12(23.1)$ & 0 & & \\
MRI & & & & \\
No avulsion & $9(17.3)$ & $18(85.7)$ & 11.83 & 0.002 \\
Unilateral avulsion & $23(44.2)$ & $3(14.3)$ & & \\
Bilateral avulsion & $20(38.5)$ & 0 & & \\
\hline
\end{tabular}

3D-TPUS, 3-dimensional transperineal ultrasound; MRI, magnetic resonance imaging; UpfmC, underactive pelvic floor muscle contraction; NpfmC, normal pelvic floor muscle contraction. 
3D-TPUS and $<7.6 \%$ on MRI could predict UpfmC with a sensitivity of $46.2 \%$ and $82.7 \%$, respectively and a specificity of $100 \%$ and $85.7 \%$, respectively. Furthermore, a cut-off percentage decrease in

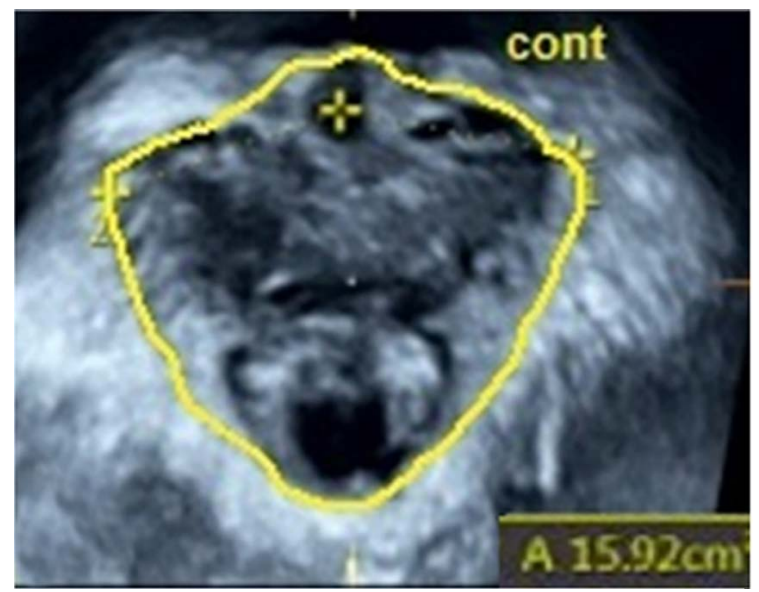

A

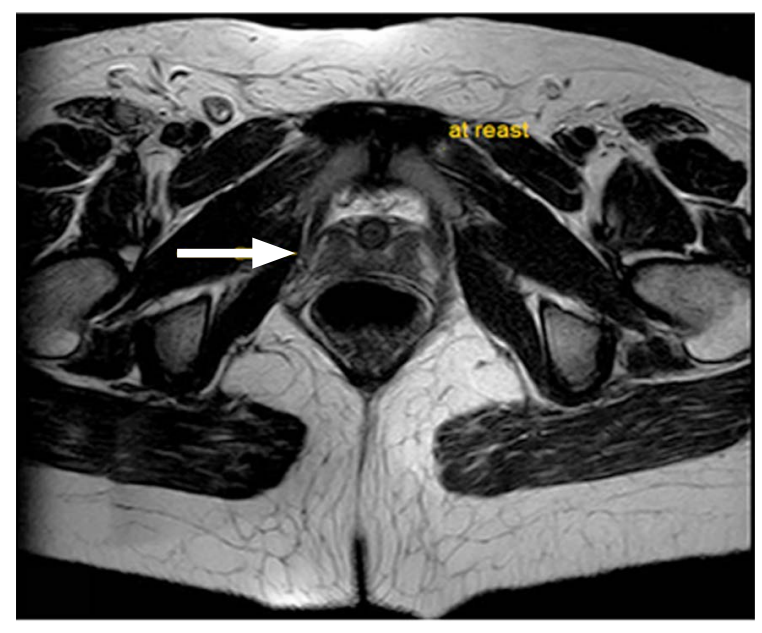

B

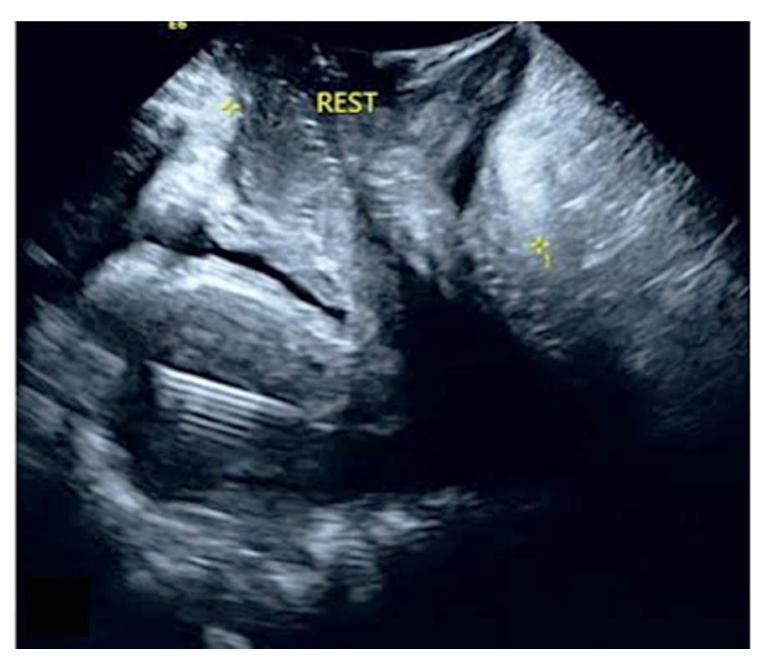

D
LH area of $<3.4 \%$ on 3D-TPUS and $<3.8 \%$ on MRI could predict UpfmC with a sensitivity of $75.0 \%$ and $88.5 \%$, respectively and a specificity of $81.0 \%$ and $95.8 \%$, respectively. When comparing the

Fig. 2. A 29-year-old primipara woman with pelvic lump and dyspareunia.

Digital palpation revealed rectocele with no avulsion and a Modified Oxford Score grade of 3. A. Three-dimensional transperineal ultrasonography (TPUS) at contraction revealed no avulsion (hiatal area $=15.92 \mathrm{~cm}^{2}$ ). B, C. Magnetic resonance imaging axial cuts show laxity and signal change in the right puborectalis muscle, denoting partial avulsion (arrow in B) with a reduction in the hiatal area from $16.13 \mathrm{~cm}^{2}$ at rest (B) to $15.69 \mathrm{~cm}^{2}$ at contraction (C). D, E. Sagittal 3-dimensional TPUS shows a reduction in the levator hiatal antero-posterior diameter from $67.5 \mathrm{~mm}$ at rest (D) to $62.9 \mathrm{~mm}$ at contraction (E).

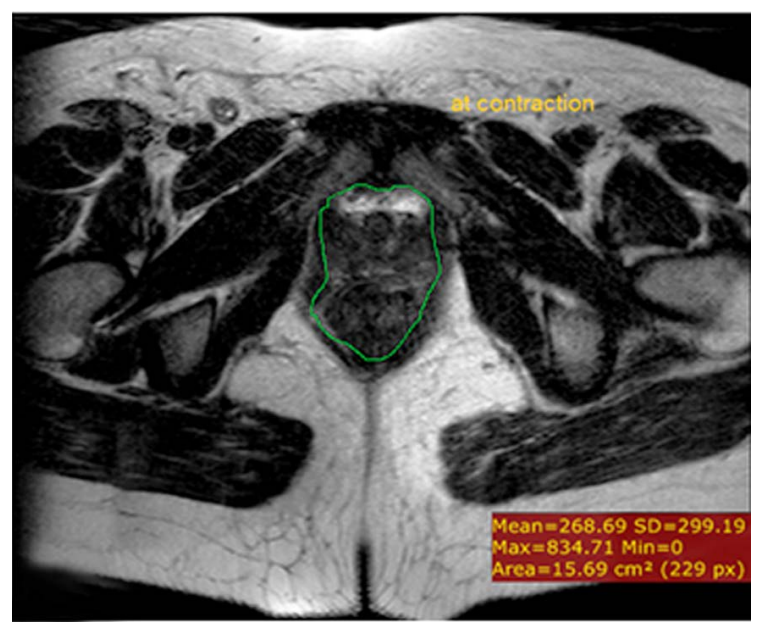

C

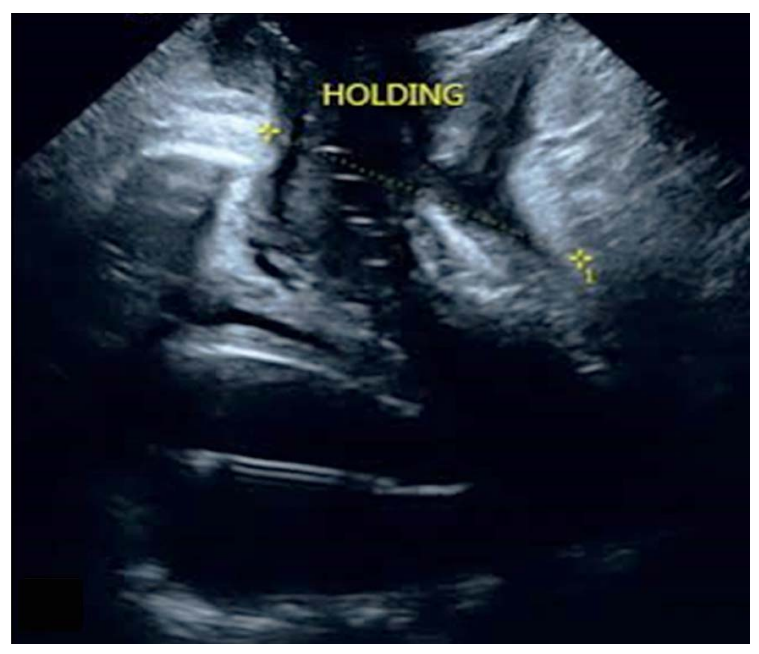

E 


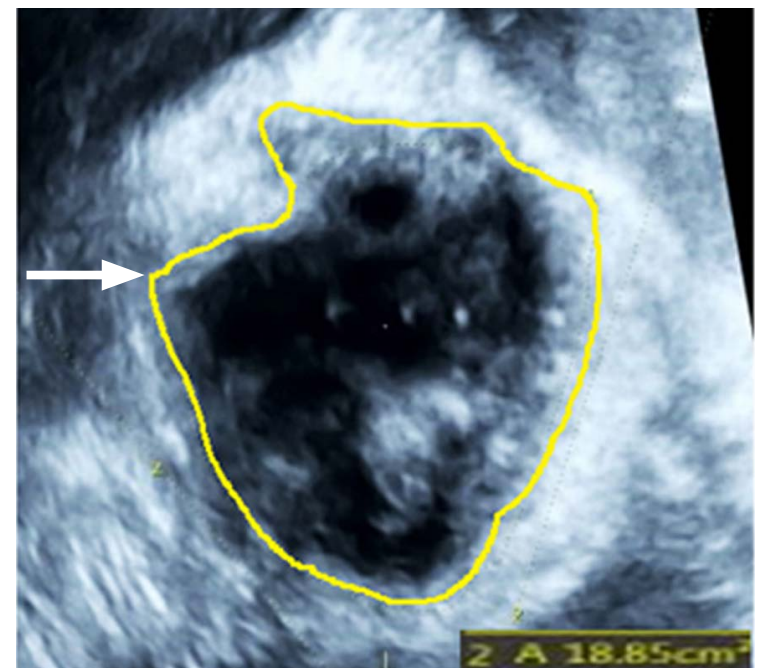

A

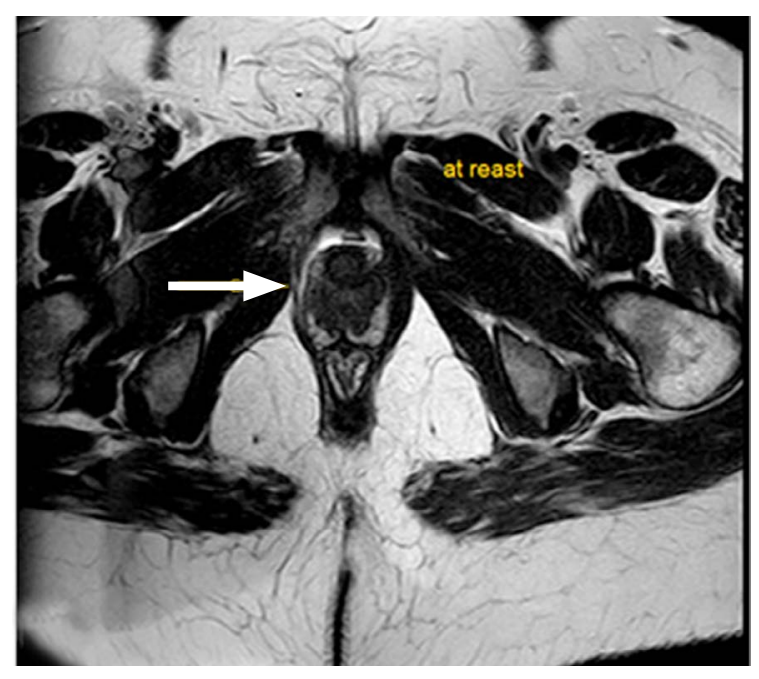

B

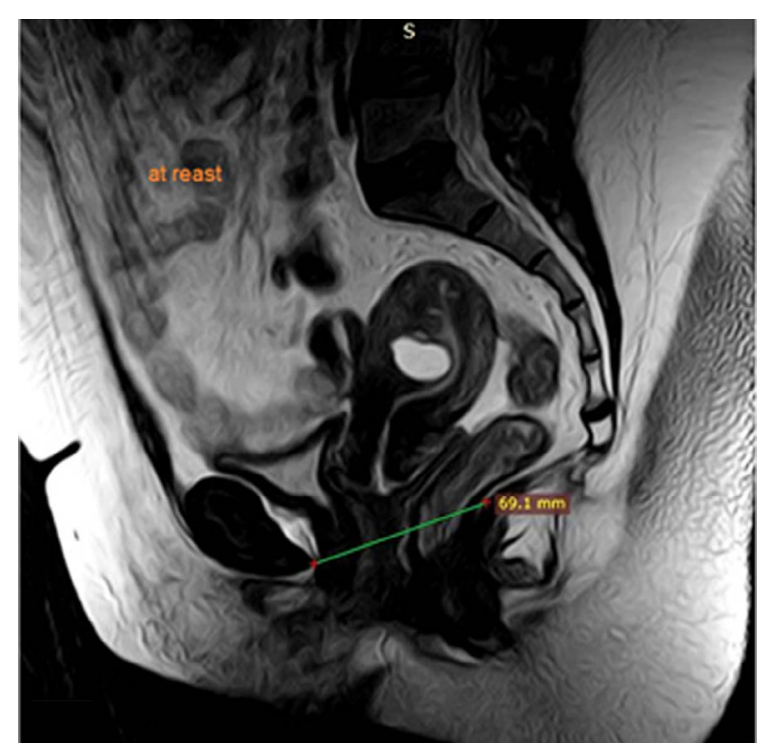

D
Fig. 3. A 44-year-old multipara (4 births) woman with a pelvic lump, urinary incontinence, and dyspareunia.

Digital palpation revealed rectocele and cystocele with no avulsion and a Modified Oxford Score grade of 3. A. Threedimensional transperineal ultrasonography at contraction reveals avulsion (arrow) of the right puborectalis muscle (hiatal area $=18.85 \mathrm{~cm}^{2}$ ). B, C. Magnetic resonance imaging (MRI) axial cuts show complete avulsion of the right puborectalis muscle (arrow in $\mathrm{B}$ ) with thinning and fatty changes at rest (B) and contraction (C). D, E. MRI sagittal cuts show a reduction in the levator hiatal anteroposterior diameter from $69.1 \mathrm{~mm}$ at rest (D) to $66.4 \mathrm{~mm}$ at contraction (E).

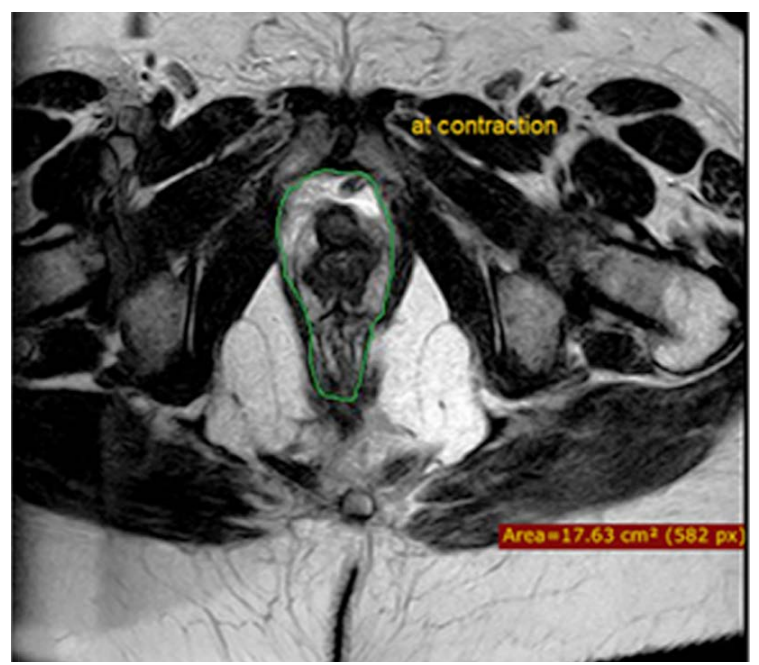

C

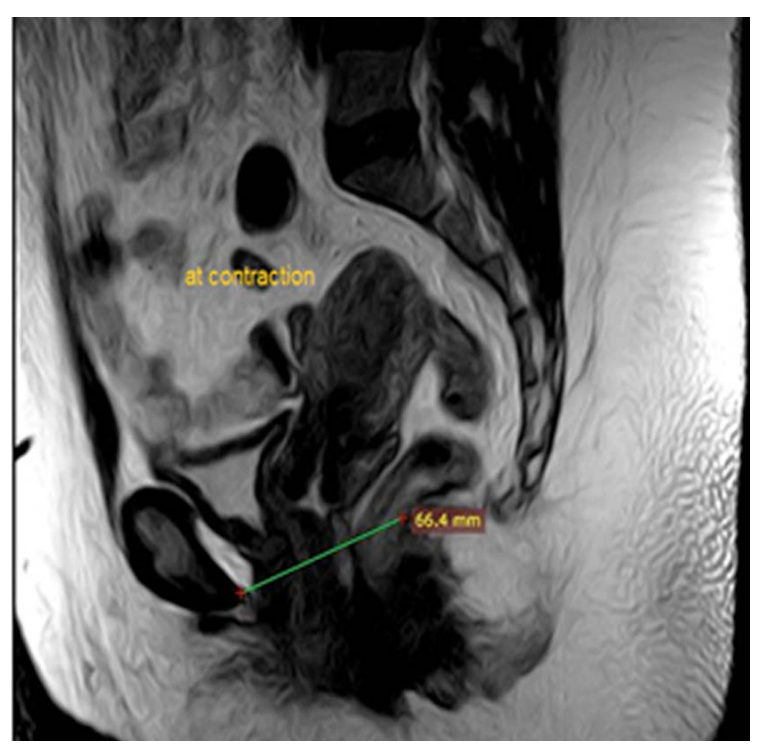

E 
ROC curves, it was found that the MRI percentage decrease in $\mathrm{LH}$ area was more sensitive and specific than that of 3D-TPUS for the prediction of UpfmC (Table 6, Fig. 4).

Table 4. Percentage changes in LHap diameter and LH area measured by 3D-TPUS and MRI from rest to contraction in both groups

\begin{tabular}{lccc}
\hline \multicolumn{1}{c}{ Measurement } & UpfmC $(\mathrm{n}=52)$ & NpfmC $(\mathrm{n}=21)$ & P-value \\
\hline 3D-TPUS & & & \\
LHap at rest $(\mathrm{mm})$ & $77.51 \pm 11.23$ & $66.51 \pm 10.33$ & $<0.001$ \\
LHap at contraction $(\mathrm{mm})$ & $68.23 \pm 8.72$ & $54.39 \pm 12.10$ & $<0.001$ \\
Mean decrease $(\%)$ & $10.51 \pm 7.14$ & $18.57 \pm 5.18$ & $<0.001$ \\
LH area at rest $\left(\mathrm{cm}^{2}\right)$ & $17.92 \pm 1.86$ & $15.83 \pm 1.27$ & $<0.001$ \\
LH area at contraction $\left(\mathrm{cm}^{2}\right)$ & $17.53 \pm 1.29$ & $14.20 \pm 2.11$ & $<0.001$ \\
Mean decrease $(\%)$ & $4.07 \pm 1.40$ & $10.19 \pm 3.51$ & $<0.001$ \\
MRI & & & \\
LHap at rest $(\mathrm{mm})$ & $66.70 \pm 3.12$ & $61.63 \pm 1.01$ & $<0.001$ \\
LHap at contraction $(\mathrm{mm})$ & $59.57 \pm 4.30$ & $51.50 \pm 2.09$ & $<0.001$ \\
Mean decrease $(\%)$ & $8.08 \pm 4.61$ & $14.41 \pm 3.38$ & $<0.001$ \\
LH area at rest $\left(\mathrm{cm}^{2}\right)$ & $17.14 \pm 1.09$ & $15.37 \pm 0.65$ & $<0.001$ \\
LH area contraction $\left(\mathrm{cm}^{2}\right)$ & $16.63 \pm 1.77$ & $13.18 \pm 0.74$ & $<0.001$ \\
Mean decrease $(\%)$ & $2.59 \pm 2.20$ & $3.55 \pm 3.30$ & $<0.001$ \\
\hline
\end{tabular}

LHap diameter, levator hiatal antero-posterior diameter; LH area, levator hiatal area; 3D-TPUS, 3-dimensional transperineal ultrasound; MRI, magnetic resonance imaging; UpfmC, underactive pelvic floor muscle contraction; NpfmC, normal pelvic floor muscle contraction.

Table 5. Spearman rank correlation coefficients between MOS and the measurements made on 3D-TPUS and MRI for the assessment of PFM contraction

\begin{tabular}{lll}
\hline \multicolumn{1}{c}{ Assessment method } & $\begin{array}{c}\text { Correlation } \\
\text { coefficient (rs) }\end{array}$ & P-value \\
\hline 3D-TPUS & & \\
Reduction in LHap diameter, mean (mm) & 0.76 & $<0.001$ \\
Reduction in LHap diameter (\%) & 0.80 & $<0.001$ \\
Reduction in LH area, mean ( $\left.\mathrm{cm}^{2}\right)$ & 0.67 & $<0.001$ \\
Reduction in LH area (\%) & 0.60 & $<0.001$ \\
MRI & & \\
Reduction in LH area, mean ( $\left.\mathrm{cm}^{2}\right)$ & 0.66 & $<0.001$ \\
Reduction in LH area (\%) & 0.70 & $<0.001$ \\
Reduction in LHap diameter, mean (mm) & 0.68 & $<0.001$ \\
Reduction in LHap diameter (\%) & 0.82 & $<0.001$ \\
\hline
\end{tabular}

$r$-value: $r=-1$, perfect disagreement; $r_{s}=0$, independent ranking; $r_{s}=+1$, perfect agreement.

MOS, Modified Oxford Score; 3D-TPUS, 3-dimensional transperineal ultrasound; MRI, magnetic resonance imaging; PFM, pelvic floor muscle; LHap diameter, levator hiatal antero-posterior diameter; $\mathrm{LH}$ area, levator hiatal area.
Table 6. Predictive values of the percentage decrease in LHap diameter and LH area measured by 3D-TPUS and MRI in the diagnosis of UpfmC

\begin{tabular}{lcccc}
\hline & $\begin{array}{c}\text { Cut-off } \\
\text { decrease (\%) }\end{array}$ & AUC & $\begin{array}{c}\text { Sensitivity } \\
(\%)\end{array}$ & $\begin{array}{c}\text { Specificity } \\
(\%)\end{array}$ \\
\hline 3D-TPUS & & & & \\
LHap diameter & $<6.5$ & 0.64 & 46.2 & 100 \\
LH area & $<3.4$ & 0.77 & 75.0 & 81.0 \\
MRI & & & & \\
LHap diameter & $<7.6$ & 0.84 & 82.7 & 85.7 \\
LH area & $<3.8$ & 0.90 & 88.5 & 95.8 \\
\hline
\end{tabular}

LHap diameter, levator hiatal antero-posterior diameter; LH area, levator hiatal area; 3D-TPUS, 3-dimensional transperineal ultrasound; MRI, magnetic resonance imaging; UpfmC, underactive pelvic floor muscle contraction; AUC, area under the curve.

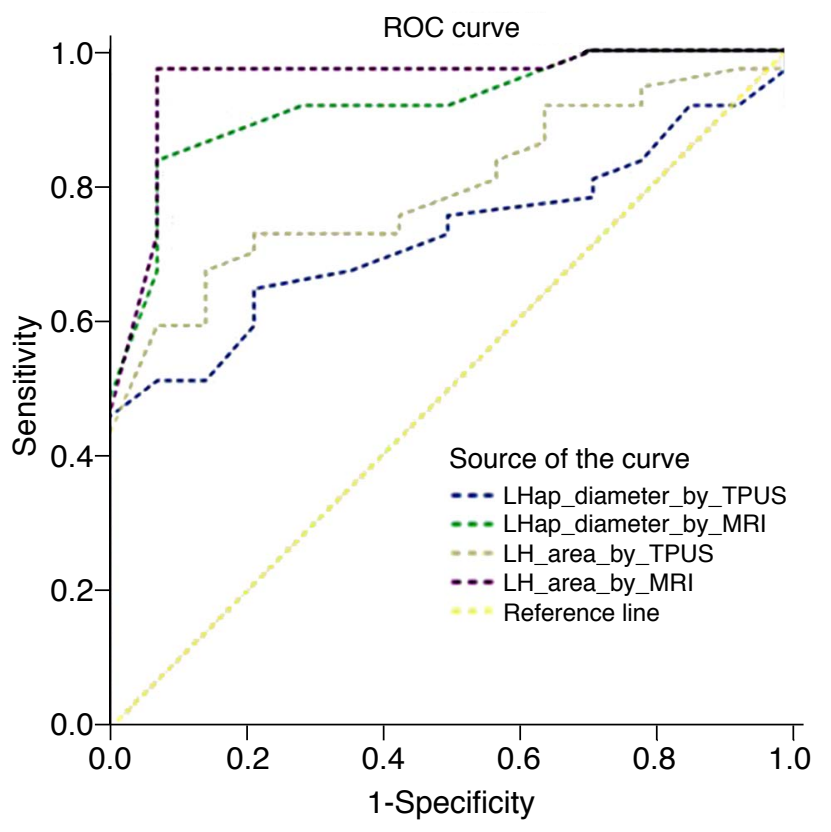

Diagonal segments are produced by ties

Fig. 4. Receiver operating characteristic (ROC) curve analysis of the percentage decrease of levator hiatal antero-posterior (LHap) diameter and levator hiatal (LH) area on 3-dimensional transperineal ultrasonography (3D-TPUS) and magnetic resonance imaging (MRI). The graph shows that the LHap diameter on 3D-TPUS has a cutoff value of $<6.5 \%$ (area under the curve [AUC], 0.64 ; sensitivity, $46.2 \%$; specificity, $100 \%)$, the LHap diameter on MRI has a cut-off value of $<7.6 \%$ (AUC, 0.84 ; sensitivity, $82.7 \%$; specificity, $85.7 \%)$, the LH area by 3D-TPUS has a cut-off value of $<3.4 \%$ (AUC, 0.77; sensitivity, 75\%; specificity, $81 \%$ ), and the LH area by MRI has a cut-off value of $<3.8 \%$ (AUC, 0.9 ; sensitivity, $88.5 \%$; specificity, $95.8 \%$ ). 


\section{Discussion}

In the current study, we assessed PFM morphology and function using both 3D-TPUS and MRI in women with PFD symptoms and compared imaging findings to clinical findings. Our results confirmed that the reduction in LHap diameter and LH area on 3D-TPUS and MRI were objective parameters indicating PFM contraction. The proportional changes in women with UpfmC were significantly lower than those in women with NpfmC. For the reduction in the LHap diameter and $\mathrm{LH}$ area at maximum PFM contraction, statistically significant positive correlations $(P<0.001)$ were found between both 3D-TPUS and MRI and digital palpation. This means that either 3D-TPUS or MRI can be employed together with digital palpation to assess PFM contraction. These results are comparable to those of previous studies [13-16]. Van Delft et al. [13] investigated pregnant and puerperal women using TPUS and found percentage decreases of $16.0 \% \pm 8.9 \%$ for LHap and $16.6 \% \pm 12.9 \%$ for hiatal area. Volloyhaug et al. [14] used perineometry together with TPUS and found a change in LHap diameter of $<7 \%$ in women with absent contractions on palpation, $7 \%-18 \%$ in those with weak contractions, and $18 \%-35 \%$ in those with normal contractions. However, perineometry is rarely used in gynecology. Dietz et al. [15] reported that the maximum reduction in LHap diameter was only $7 \%$ in women with UpfmC using 3D-TPUS.

We found a strong correlation between MOS scores and the percentage reduction in LHap diameter $(r=0.80$ and $r=0.82$ on 3D-TPUS and MRI, respectively), in agreement with Volloyhaug et al. [14] ( $r=0.69)$. Additionally, Van Delft et al. [13] found a good correlation $(r=0.51)$, but they used bladder neck movement during pelvic floor muscle contraction. However, both of those studies only used US.

The MRI percentage decrease in $\mathrm{LH}$ area, with a cut-off value of $3.8 \%$, was more sensitive and specific than that of 3D-TPUS (3.4\%) for predicting UpfmC, which can be explained by the higher soft tissue resolution of MRI in delineating anatomical landmarks, as well as by the absence of motion artifacts during US examinations. To our knowledge, no previous studies have identified cut-offs for $\mathrm{LH}$ area and LHap diameter measurements on US or MRI that can predict UpfmC. However, in our study, the mean LH area measurements at rest and during contraction were similar to those reported in other studies $[16,17]$ that evaluated LH area in patients with UpfmC.

Regarding morphological aspects, we diagnosed levator avulsion in $27.1 \%$ of cases using TPUS. However, MRI raised this percentage to $63.4 \%$, as MRI can detect subtle partial trauma as signal intensity changes, laxity, or asymmetrical thinning in the muscle. Our US detection rate was similar to that found by Shek et al. [17], who detected levator avulsion in $27.0 \%$ of women seen within 1.9 months after primary repair of obstetric anal sphincter tears. Our findings were also similar to those of Dietz et al. [18], who found levator avulsion in $30 \%$ of patients with symptoms and signs of POP using tomographic US imaging. Major PFM defects were diagnosed using 3D-TPUS in 21\%-37\% of patients by Abdool et al. [16] and in $34 \%$ by Thompson et al. [19].

We found significant positive relationships among age, BMI, vaginal delivery, parity, and UpfmC, which is supported by Greer et al. [20], who noted that the increased intra-abdominal pressure in patients with increased sagittal abdominal diameter and BMI stressed the pelvis, causing damage.

We found that poor intrinsic urethral function was the main cause of SUI, not weak PFM $(P=0.220)$, which is similar to the findings of DeLancey et al. [21]. However, our findings are in contrast to those of Shek et al. [17], who reported levator injury in women suffering from SUI and supported those findings by the proven efficacy of PFM training in the treatment of SUI. We did not find an association between PFD and FI $(P=0.450)$, although such an association was reported by Dietz et al. [18] using a perineal dynamometer.

Finally, palpation provides information such as muscle resting tone and points of pain or reduced sensation. However, training is necessary for palpation, and it relies on the comparison of findings with a supposedly intact contralateral side for the diagnosis of avulsion, making bilateral defects much harder to detect. 3D-TPUS is more widely available and less expensive than MRI. Moreover, the stored volumes can be used as guidance for the interpretation of digital palpation measurements and in patient education about how to contract the PFM. Therefore, it can be used as an initial objective tool for the evaluation of PFM contraction. MRI has superior softtissue resolution and can be used to diagnose PFD in patients with multi-compartment involvement, but it is less accessible. Hence, its use can be limited to doubtful cases and cases of recurrence after surgery.

Our study has several strengths. It is a prospective study, which avoids the selection bias of retrospective studies. The MOS was used, which is a validated method to assess pelvic floor strength. However, our study also has limitations. First, the size of the study population was limited. Second, we did not include measurements during the Valsalva maneuver as we aimed to detect early cases. Third, digital palpation is a limited reference standard, as it is operatordependent. However, we strengthened the validity of the digital palpation findings by using an expert panel. Finally, no comparison was made with asymptomatic PFD patients to define cut-offs.

In conclusion, 3D-TPUS and MRI had a strong positive correlation with palpation, and at certain cut-offs for hiatal dimensions, they can be used as objective tools to improve the accuracy of PFD diagnosis and to help in management planning. This study could 
be used as a source of preliminary evidence to determine which patients can benefit from pelvic training to improve PFM function and which need surgery using the cut-offs for percentage decrease.

ORCID: Mohammad Abd Alkhalik Basha: https://orcid.org/0000-0002-9075-8020

\section{Author Contributions}

Conceptualization: Basha MAA, Madkour NM, Al-Molla RM, Tantwy EF, El-Haieg DO. Data acquisition: Madkour NM, Ahmad RA, Sadek SM, Almassry HN, Altaher KM, Mahmoud NEM. Data analysis interpretation: Basha MAA, Madkour NM, Al-Molla RM, Aly SA, Tantwy EF. Drafting of the manuscript: Basha MAA, Madkour NM, Al-Molla RM, El-Haieg DO. Critical revision of the manuscript: Basha MAA, Madkour NM, Al-Molla RM, El-Haieg DO. Approval of the final version of the manuscript: all authors.

\section{Conflict of Interest}

No potential conflict of interest relevant to this article was reported.

\section{References}

1. Voorham-van der Zalm PJ, Lycklama a Nijeholt GA, Elzevier HW, Putter H, Pelger RC. "Diagnostic investigation of the pelvic floor": a helpful tool in the approach in patients with complaints of micturition, defecation, and/or sexual dysfunction. J Sex Med 2008;5:864-871.

2. Santoro GA, Wieczorek AP, Dietz HP, Mellgren A, Sultan AH, Shobeiri SA, et al. State of the art: an integrated approach to pelvic floor ultrasonography. Ultrasound Obstet Gynecol 2011;37:381396.

3. Reisenauer C, Muche-Borowski C, Anthuber C, Finas D, Fink T, Gabriel B, et al. Interdisciplinary S2e guideline for the diagnosis and treatment of stress urinary incontinence in women: short version AWMF Registry No. 015-005, July 2013. Geburtshilfe Frauenheilkd 2013;73:899-903.

4. Dietz HP, Shek C. Levator avulsion and grading of pelvic floor muscle strength. Int Urogynecol J Pelvic Floor Dysfunct 2008; 19:633-636.

5. Dietz HP, Wilson PD, Clarke B. The use of perineal ultrasound to quantify levator activity and teach pelvic floor muscle exercises. Int Urogynecol J Pelvic Floor Dysfunct 2001;12:166-168.

6. Dietz HP, Shek KL. Levator defects can be detected by 2D translabial ultrasound. Int Urogynecol J Pelvic Floor Dysfunct 2009;20:807811.

7. Kelvin FM, Hale DS, Maglinte DD, Patten BJ, Benson JT. Female pelvic organ prolapse: diagnostic contribution of dynamic cystoproctography and comparison with physical examination. AJR
Am J Roentgenol 1999;173:31-37.

8. Dietz HP, Abbu A, Shek KL. The levator-urethra gap measurement: a more objective means of determining levator avulsion? Ultrasound Obstet Gynecol 2008;32:941-945.

9. Dietz HP. Ultrasound imaging of the pelvic floor. Part II: threedimensional or volume imaging. Ultrasound Obstet Gynecol 2004;23:615-625.

10. Dietz HP, Shek C, Clarke B. Biometry of the pubovisceral muscle and levator hiatus by three-dimensional pelvic floor ultrasound. Ultrasound Obstet Gynecol 2005;25:580-585.

11. Steensma $A B$, Konstantinovic $M L$, Burger $C W$, de Ridder $D$, Timmerman $D$, Deprest J. Prevalence of major levator abnormalities in symptomatic patients with an underactive pelvic floor contraction. Int Urogynecol J 2010;21:861-867.

12. Hajian-Tilaki K. Sample size estimation in diagnostic test studies of biomedical informatics. J Biomed Inform 2014;48:193-204.

13. van Delft $K$, Thakar R, Sultan AH. Pelvic floor muscle contractility: digital assessment vs transperineal ultrasound. Ultrasound Obstet Gynecol 2015;45:217-222.

14. Volloyhaug I, Morkved S, Salvesen O, Salvesen KA. Assessment of pelvic floor muscle contraction with palpation, perineometry and transperineal ultrasound: a cross-sectional study. Ultrasound Obstet Gynecol 2016;47:768-773.

15. Dietz HP, Gillespie AV, Phadke P. Avulsion of the pubovisceral muscle associated with large vaginal tear after normal vaginal delivery at term. Aust N Z J Obstet Gynaecol 2007;47:341-344.

16. Abdool Z, Shek KL, Dietz HP. The effect of levator avulsion on hiatal dimension and function. Am J Obstet Gynecol 2009;201:89.e1-89. e5.

17. Shek KL, Guzman-Rojas R, Dietz HP. Residual defects of the external anal sphincter following primary repair: an observational study using transperineal ultrasound. Ultrasound Obstet Gynecol 2014;44:704-709.

18. Dietz HP, Bernardo MJ, Kirby A, Shek KL. Minimal criteria for the diagnosis of avulsion of the puborectalis muscle by tomographic ultrasound. Int Urogynecol I 2011;22:699-704.

19. Thompson JA, O'Sullivan PB, Briffa NK, Neumann P. Assessment of voluntary pelvic floor muscle contraction in continent and incontinent women using transperineal ultrasound, manual muscle testing and vaginal squeeze pressure measurements. Int Urogynecol J Pelvic Floor Dysfunct 2006;17:624-630.

20. Greer WJ, Richter HE, Bartolucci AA, Burgio KL. Obesity and pelvic floor disorders: a systematic review. Obstet Gynecol 2008;112:341349.

21. DeLancey JO, Trowbridge ER, Miller JM, Morgan DM, Guire K, Fenner $D E$, et al. Stress urinary incontinence: relative importance of urethral support and urethral closure pressure. J Urol 2008;179: 2286-2290. 